\title{
PROPERTIES OF THE SOLUTIONS OF THE CAUCHY PROBLEM FOR THE (CLASSICAL) COUPLED MAXWELL-DIRAC EQUATIONS IN ONE SPACE DIMENSION
}

\author{
ROBERT T. GLASSEY AND JOHN M. CHADAM ${ }^{1}$
}

\begin{abstract}
Solutions of the Maxwell-Dirac equations coupled through the standard electromagnetic interaction are shown to blow up at each spatial point for large times. This is used to show that these solutions do not tend asymptotically to free solutions. In addition it is used to prove that these equations do not admit a nontrivial stationary solution.
\end{abstract}

1. Introduction. The Cauchy problem for the coupled Maxwell-Dirac equations

$$
\begin{gathered}
\left(-i \gamma^{\mu} \partial_{\mu}+m\right) \psi=g \gamma^{\mu} v_{\mu} \psi, \\
\square v_{\mu}=\left(\partial_{x}^{2}-\partial_{0}^{2}\right) v_{\mu}=-g \bar{\psi} \gamma_{\mu} \psi, \\
\partial^{\mu} v_{\mu}=0
\end{gathered}
$$

has recently been treated in one space dimension [1]. (See this article and [2] for the notation and extended comments on the formulation of the problems treated in this work.)

In particular it has been shown that a unique generalized solution of equations (1) possessing one $L^{2}$-derivative exists for all time. Here we investigate the asymptotic behavior of this solution. Specifically we will show that the solution blows up at each spatial point as $|t|$ becomes infinite. This behavior allows us then to establish two rather different results; namely, these solutions do not scatter nor are they stationary in the sense of Berger [3]. The main blow up theorem follows by means of a simple calculation from the classical form of the solution to the wave equation in one dimension. For this reason we first prove a regularity theorem for the generalized solutions of reference [1].

Received by the editors June 4, 1973.

AMS (MOS) subject classifications (1970). Primary 35F25, 35B40; Secondary 35Q99, $81 \mathrm{~A} 39$.

Key words and phrases. Maxwell-Dirac equations, Cauchy problem, regularity, blowup, scattering, stationary solutions.

${ }^{1}$ Both authors were supported in part by NSF GP 37630.

(c) American Mathematical Society 1974 
2. Regularity. We begin by setting the notation and briefly summarizing the work in reference [1]. In one space dimension the spinor field $\psi$ and the real-valued vector field $v$ have two components. The (integrated form of the) equivalent vector-valued version of equations (1a) and (1b) is

$$
\begin{gathered}
\psi(t)=D\left(t-t_{0}\right) \psi^{0}+g \int_{t_{0}}^{t} D(t-s) V(s) \psi(s) d s, \\
\left(\begin{array}{c}
v(t) \\
\dot{v}(t)
\end{array}\right)=M\left(t-t_{0}\right)\left(\begin{array}{c}
v^{0} \\
\dot{v}^{0}
\end{array}\right)+g \int_{t_{0}}^{t} M(t-s)\left(\begin{array}{c}
0 \\
J(s)
\end{array}\right) d s,
\end{gathered}
$$

where $\left(\psi^{0},\left(v^{0}, \dot{v}^{0}\right)^{\mathrm{T}}\right)^{2}$ is the Cauchy data at time $t_{0}, \dot{v}=d v / d t, D(\cdot)$ and $M(\cdot)$ are the free Dirac and wave propagators, and

and

$$
V(t)=i\left(v_{0}(t)+v_{1}(t) \gamma^{0} \gamma^{1}\right) \quad \text { if } \quad v(t)=\left(\begin{array}{l}
v_{0}(t) \\
v_{1}(t)
\end{array}\right)
$$

$$
J(t)=\left(\begin{array}{c}
J_{0}(t) \\
J_{1}(t)
\end{array}\right)=\left(\begin{array}{c}
\psi^{\dagger} \psi(t) \\
-\psi^{\dagger} \gamma^{0} \gamma^{1} \psi(t)
\end{array}\right)
$$

where $\psi^{\dagger}$ is the conjugate transpose of $\psi$. The existence theorem proved in [1] is: For given data $\left(\psi^{0},\left(v^{0}, \dot{v}^{0}\right)^{\mathrm{T}}\right) \in H^{\mathbf{1}} \oplus\left(H^{1} \oplus L^{2}\right),{ }^{3}$ there exist unique functions $\psi$ and $v$ with

$$
t \rightarrow\left(\psi(t),\left(\begin{array}{c}
v(t) \\
\dot{v}(t)
\end{array}\right)\right):\left(t_{0}, \infty\right) \rightarrow H^{1} \oplus\left(H^{1} \oplus L^{2}\right)
$$

continuous, which for all $t_{0}<t<\infty$ satisfy equations (2). Classical solutions of equations will be obtained as a corollary of the following result.

THeORem 2.1. The Cauchy problem for equations (2) has a unique solution in $H^{n} \oplus\left(H^{n} \oplus H^{n-1}\right)$ for each $n$.

Proof. The argument consists in showing that the general results of Segal [4] can be applied. In particular we must show that the map

$$
\left(\psi,\left(\begin{array}{l}
v \\
\dot{v}
\end{array}\right)\right) \rightarrow\left(V \psi,\left(\begin{array}{c}
0 \\
J
\end{array}\right)\right): H^{n} \oplus\left(H^{n} \oplus H^{n-1}\right) \rightarrow H^{n} \oplus\left(H^{n} \oplus H^{n-1}\right)
$$

is locally Lipschitzian in order to obtain the local existence theorem and

${ }^{2}$ Throughout, ${ }^{\mathrm{T}}$ will denote the transpose of the vector.

${ }^{3}$ Actually, the spaces $D_{1} \oplus M_{1 / 2}$ used in [1] are equivalent to $H^{1} \oplus\left(H^{1} \oplus L^{2}\right)$. For our present purposes it is more convenient to use the Sobolev norm and notation. Here and throughout the rest we shall use the same notation for a space and its two-fold direct sum. 
then establish that the solution can be extended for all time by showing that its $H^{n} \oplus\left(H^{n} \oplus H^{n-1}\right)$-norm remains finite [4, Theorem 1, p. 343]. The calculational aspects of both of these problems are summarized in the following lemma (which is true in arbitrary dimension for $k \geqq n / 2$, $n$ odd, $k>n / 2, n$ even).

Lemma 2.2. Suppose $f, h \in H^{k}\left(E^{1}\right)$; then for $k \geqq 1$

$$
\left\|D^{k}(f h)\right\|_{2} \leqq \text { const }\|f\|_{k, 2}\|h\|_{k, 2},
$$

where $D$ is the strong- $L^{2}$ derivative.

Proof of Lemma. Using the Leibniz formula for strong derivatives, $D^{k}(f h)$ consists of a linear combination of terms $D^{l} f D^{m} h$ with $l+m=k$. Now Sobolev inequalities give that $\left\|D^{l} f\right\|_{p} \leqq$ const $\left\|D^{k} f\right\|_{2}^{\gamma}\|f\|_{2}^{1-\gamma}$ where $l k^{-1} \leqq \gamma \leqq 1$ and $p^{-1}=l+\gamma\left(\frac{1}{2}-k\right)+(1-\gamma) / 2$. Likewise,

$$
\left\|D^{m} h\right\|_{q} \leqq \text { const }\left\|D^{k} h\right\|_{2}^{\delta}\|h\|_{2}^{1-\delta}
$$

with $m k^{-1} \leqq \delta \leqq 1$ and $q^{-1}=m+\delta\left(\frac{1}{2}-k\right)+(1-\delta) / 2$. All that remains is to show that $\frac{1}{2}=p^{-1}+q^{-1}=m+l+1-(\gamma+\delta) k$ can be satisfied with $\gamma, \delta$ in the required range. The last is equivalent to $\gamma+\delta=\left(k+\frac{1}{2}\right) k^{-1}$ which obtains if $\gamma=\left(l+\frac{1}{4}\right) k^{-1}$ and $\delta=\left(m+\frac{1}{4}\right) k^{-1}$. This choice is in the acceptable range provided that $l$ and $m \leqq k-1$. If $l$ or $m=k$ then $\left\|f D^{k} h\right\|_{2} \leqq\|f\|_{\infty}\left\|D^{k} h\right\|_{2}$ replaces the above in view of the Sobolev inequality

$$
\|f\|_{\infty} \leqq \text { const }\|f\|_{2}^{1 / 2}\|D f\|_{2}^{1 / 2}
$$

for $f \in H^{1}\left(E^{1}\right)$. The proof of the lemma is completed by noticing that

$$
\left\|D^{k} f\right\|_{2}^{\gamma}\|f\|_{2}^{1-\gamma} \leqq \gamma\left\|D^{k} f\right\|_{2}+(1-\gamma)\|f\|_{2} \leqq 2\|f\|_{k, 2} .
$$

Returning to the proof of the theorem, the Lipschitz property follows from the above because every nonzero component of the nonlinearity is the sum of products of the components of the spinor and vector field. Thus, for example, $D^{k}\left(i v_{0} \psi_{1}-i \tilde{v}_{0} \tilde{\psi}_{1}\right)$ is written as the sum of terms like

$$
i\left[D^{l}\left(v_{0}-\tilde{v}_{0}\right) D^{m} \psi_{1}+D^{l} v_{0} D^{m}\left(\psi_{1}-\tilde{\psi}_{1}\right)\right]
$$

and estimated as in the lemma.

The extension to all time in $H^{n} \oplus\left(H^{n} \oplus H^{n-1}\right)$ is, on the other hand, a continuation of the "boot-strap" begun in [1]. Specifically, we show that the $H^{n} \oplus\left(H^{n} \oplus H^{n-1}\right)$-norm of the solution remains finite by induction. The first step is the content of reference [1]. For the inductive step assume

$$
\|\psi(t)\|_{n-1,2}+\|v(t)\|_{n-1,2}+\|\dot{v}(t)\|_{n-2,2}=f_{n-1}(t)
$$


is finite for each $t_{0} \leqq t<\infty$. Then, from equation (2b),

$$
\left\|\left(\begin{array}{c}
v(t) \\
\dot{v}(t)
\end{array}\right)\right\|_{n \oplus n-1,2} \leqq c\left(1+\left(t-t_{0}\right)\right)\left\|\left(\begin{array}{c}
v^{0} \\
\dot{v}^{0}
\end{array}\right)\right\|_{n \oplus n-1,2}
$$

using the estimate

$$
+c g \int_{t_{0}}^{t}(1+(t-s))\left\|\left(\begin{array}{c}
0 \\
J(s)
\end{array}\right)\right\|_{n \oplus n-1,2} d s,
$$

$$
\left\|M(t)\left(\begin{array}{l}
\varphi_{1} \\
\varphi_{2}
\end{array}\right)\right\|_{n \oplus n-1,2} \leqq c(n)(1+t)\left\|\left(\begin{array}{l}
\varphi_{1} \\
\varphi_{2}
\end{array}\right)\right\|_{n \oplus n-1,2} .
$$

But

$$
\left\|\left(\begin{array}{c}
0 \\
J(s)
\end{array}\right)\right\|_{n \oplus n-1,2}=\left\|\psi^{\dagger} \psi(s)\right\|_{n-1,2} \leqq \text { const }\|\psi(s)\|_{n-1,2}^{2} \leqq \text { const } f_{n-1}^{2}(s) .
$$

Thus $\left\|(v(t), \dot{v}(t))^{\mathrm{T}}\right\|_{n \oplus n-1,2}$ is continuous and finite for each $t_{0} \leqq t<\infty$. Likewise, from equation (2a),

$$
\begin{aligned}
\|\psi(t)\|_{n, 2} & \leqq c\left\|\psi^{0}\right\|_{n, 2}+g c \int_{t_{0}}^{t}\|V(s) \psi(s)\|_{n, 2} d s \\
& \leqq c\left\|\psi^{0}\right\|_{n, 2}+g \tilde{c} \int_{t_{0}}^{t}\left\|\left(\begin{array}{c}
v(s) \\
\dot{v}(s)
\end{array}\right)\right\|_{n \oplus n-1,2}\|\psi(s)\|_{n, 2} d s,
\end{aligned}
$$

using the fact that the Dirac propagator $D(t): H^{n} \rightarrow H^{n}$ is bounded and the estimate (3). The result now follows by applying the Gronwall lemma to inequality (5).

If the data $\psi^{0},\left(v^{0}, \dot{v}^{0}\right)^{\mathrm{T}}$ have arbitrarily many $L^{2}$-derivatives (for example, if they are in $\mathscr{S}$ ), then the solution of equations (2) is in $\bigcap_{n=1}^{\infty} H^{n} \oplus\left(H^{n} \oplus H^{n-1}\right)$ and can be differentiated an arbitrary number of times [4, Theorems 2 and 3, pp. 351-353] to give that

$$
\psi, v \in \bigcap_{n=1}^{\infty} H^{n}\left(\left(t_{0}, \infty\right) \times E^{1}\right) .
$$

By Sobolev's Imbedding Theorem $\psi, v$ have $C^{\infty}\left(\left(t_{0}, \infty\right) \times E^{1}\right)$ representatives which satisfy equations (1a) and (1b) in the classical sense.

3. Asymptotics and scattering. Throughout this section we shall take $t_{0}=0$ for notational convenience.

THEOREM 3.1. If $\psi, v=\left(v_{0}, v_{1}\right)^{\mathrm{T}}$ is a classical solution of equations (1a), (1b) with Cauchy data $\psi^{0},\left(v^{0}, \dot{v}^{0}\right)^{\mathrm{T}}$ in $\mathscr{S}$ and $\psi^{0} \not \equiv 0$, then $\lim _{|t| \rightarrow \infty} v_{0}(x, t)=\infty$ for each $x \in E^{1}$. 
Proof. If $v_{0}^{0}$ and $\dot{v}_{0}^{0}$ are the Cauchy data for $v_{0}(x, t)$ then

$$
\begin{aligned}
v_{0}(x, t)= & \frac{1}{2}\left(v_{0}^{0}(x+t)+v_{0}^{0}(x-t)\right)+\frac{1}{2} \int_{x-t}^{x+t} \dot{v}_{0}^{0}(y) d y \\
& +\frac{g}{2} \int_{0}^{t} \int_{x-(t-\tau)}^{x+(t-\tau)} \psi^{\dagger} \psi(y, \tau) d y d \tau .
\end{aligned}
$$

Thus

$$
\lim _{t \rightarrow \infty} v_{0}(x, t)=\frac{1}{2} \int_{-\infty}^{\infty} v_{0}^{0}(y) d y+\frac{g}{2} \lim _{t \rightarrow \infty} \int_{0}^{\infty} \int_{-\infty}^{\infty} \chi_{x, t} \psi^{\dagger} \psi(y, \tau) d y d \tau
$$

where $\chi_{x, t}$ is the characteristic function of the interior of the backward characteristic cone through $(x, t)$. The last term is

$$
\frac{g}{2} \int_{0}^{\infty} \int_{-\infty}^{\infty} \psi^{\dagger} \psi(y, \tau) d y d \tau
$$

by the monotone convergence theorem, and is, in turn, equal to

$$
\frac{g}{2} \int_{0}^{\infty}\left[\int_{-\infty}^{\infty} \psi^{\dagger} \psi(y, \tau) d y\right] d \tau
$$

by the Fubini-Tonelli theorem. But

$$
\int_{-\infty}^{\infty} \psi^{\dagger} \psi(y, \tau) d y=\int_{-\infty}^{\infty} \psi^{\dagger} \psi(y, 0) d y=\int_{-\infty}^{\infty} J^{0}(y) d y>0
$$

by charge conservation [1, Proposition 3.1]. Thus $v^{0}(x, t)$ blows up at each $x$ for large $t$.

The above result can, as expected, be used to show that equations (1a) and (lb) do not have a scattering theory. More specifically we show that the physical-to-free wave operators do not exist in any reasonable sense.

THEOREM 3.2. There is no free solution (i.e. solution of (1a), (1b) with $g=0)$ with Cauchy data in $\mathscr{S}$ to which the solution of Theorem 3.1 tends in $H^{1} \oplus\left(H^{1} \oplus L^{2}\right)$.

Proof. Suppose $\psi^{+},\left(v^{+}, \dot{v}^{+}\right)^{\mathrm{T}}$ is the free solution which is asymptotically similar to the given solution. Then

$$
\left\|v_{0}^{+}(t)-v_{0}(t)\right\|_{\infty} \leqq \text { const }\left\|v_{0}^{+}(t)-v_{0}(t)\right\|_{1,2} \rightarrow 0 \text { as } t \rightarrow \infty .
$$

But $\left\|v_{0}^{+}(t)\right\|_{\infty} \leqq c<\infty$ for all $t$ because it has data in $\mathscr{S}$ while $\left\|v_{0}(t)\right\|_{\infty} \rightarrow \infty$ as $t \rightarrow \infty$ by Theorem 3.1, thus giving the contradiction.

Theorem 3.1 can also be used to show that equations (1a), (1b) and (1c) cannot have stationary solutions (i.e. solutions $\psi, v$ with $|\psi(x, t)|=$ $|\psi(x, 0)|$ and $|v(x, t)|=|v(x, 0)|)$. Inasmuch as stationary solutions are 
analogous to bound states and hence extreme opposites to scattering solutions, it is interesting to note that Theorem 3.1 is instrumental in the proof of the nonexistence of both of these types of solutions.

THEOREM 3.3. There are no nontrivial stationary solutions of equations (1a), (1b) and (1c) with data in $\mathscr{S}$.

Proof. On the one hand $\lim _{t \rightarrow \infty} v_{0}(x, t)=\infty$ by Theorem 3.1. But integration of (1c) gives $v_{0}(x, t)=v_{0}(x, 0)+\int_{0}^{t} \partial v_{1}(x, \tau) / \partial x d \tau$. Both sides of the current conservation equation

$$
\frac{\partial \psi^{\dagger} \psi}{\partial t}(x, t)=-\frac{\partial}{\partial x} \psi^{\dagger} \gamma^{0} \gamma^{1} \psi(x, t)
$$

[1, Proposition 3.1] are zero since the solution is stationary (i.e. $\left.\psi^{\dagger} \psi(x, t)=\psi^{\dagger} \psi(x, 0)\right)$. Integration of the conservation equation with respect to $x$ from $-\infty$ to $x$ gives that $\psi^{\dagger} \gamma^{0} \gamma^{1} \psi(x, t) \equiv 0$ since $\psi \in \bigcap_{n=1}^{\infty} H^{n}$ and hence tends to zero as $|x| \rightarrow \infty$. Thus $v_{1}$ satisfies the free wave equation and so

$$
\begin{array}{r}
v_{0}(x, t)=v_{0}^{0}(x)-\frac{1}{2}\left[v_{1}^{0}(x-t)-v_{1}^{0}(x+t)\right] \\
-\frac{1}{2} \int_{0}^{t} \dot{v}_{1}^{0}(x-\tau)-\dot{v}_{1}^{0}(x+\tau) d \tau
\end{array}
$$

which is bounded at each $x$ when $t \rightarrow \infty$. The only way to remove this contradiction is to take $|\psi(x, 0)|=|\psi(x, t)| \equiv 0$, thus entirely eliminating the interaction.

\section{REFERENCES}

1. J. M. Chadam, Global solutions of the Cauchy problem for the (classical) coupled Maxwell-Dirac equations in one space dimension, J. Functional Analysis 13 (1973), 173-184.

2. L. Gross, The Cauchy problem for the coupled Maxwell and Dirac equations, Comm. Pure Appl. Math. 19 (1966), 1-15. MR 32 \#7932.

3. M. S. Berger, On the existence and structure of stationary states for a nonlinear Klein-Gordon equation, J. Functional Analysis 9 (1972), 249-261. MR 45 \#9014.

4. I. E. Segal, Non-iinear semi-groups, Ann. of Math. (2) 78 (1963), 339-364. MR 27 \#2879.

Department of Mathematics, Indiana University, Bloomington, Indiana 47401 